\title{
Postoperative acute kidney injury in high-risk patients undergoing major abdominal surgery ${ }^{\grave{2}}$
}

\author{
Stefano Romagnoli, MD ${ }^{\mathrm{a}, \mathrm{b}}$, Giovanni Zagli, MD ${ }^{\mathrm{a}, \mathrm{b}, *}$, Germana Tuccinardi, MD ${ }^{\mathrm{a}, \mathrm{b}}$, Lorenzo Tofani, PhD ${ }^{\mathrm{c}, \mathrm{d}}$, \\ Cosimo Chelazzi, MD ${ }^{\mathrm{a}, \mathrm{b}}$, Gianluca Villa, MD ${ }^{\mathrm{a}, \mathrm{b}}$, Fabio Cianchi, MD ${ }^{\mathrm{e}}$, Andrea Coratti, MD ${ }^{\mathrm{f}}$, \\ Angelo Raffaele De Gaudio, MD ${ }^{\mathrm{a}, \mathrm{b}}$, Zaccaria Ricci, $\mathrm{MD}^{\mathrm{g}}$ \\ a Department of Health Science, University of Florence, Florence, Italy \\ ${ }^{\mathrm{b}}$ Department of Anaesthesia and Intensive Care, Azienda Ospedaliero-Universitaria Careggi, Florence, Italy \\ c Department of Neurosciences, Psychology, Drug Research and Child Health, University of Florence, Florence, Italy \\ d Clinical Trials Coordinating Center, Istituto Toscano Tumori, Florence, Italy \\ e Unit of General and Endocrine Surgery, Department of Surgery and Translational Medicine, University of Florence, Florence, Italy \\ ${ }^{\mathrm{f}}$ Division of Oncological and Robotic General Surgery, Careggi University Hospital, Florence, Italy \\ g Pediatric Cardiac Intensive Care Unit, Department of Cardiology and Cardiac Surgery, Bambino Gesù Children's Hospital, IRCCS, Rome, Italy
}

\section{A R T I C L E I N F O}

\section{Keywords:}

Acute kidney injury

Postoperative AKI

\begin{abstract}
A B S T R A C T
Purpose: Acute kidney injury (AKI) is a frequent complication in high-risk patients undergoing major surgery and is associated with longer hospital stay, increased risk for nosocomial infection and significantly higher costs. Materials and methods: A prospective observational study exploring the incidence of AKI (AKIN classification at any stage) in high-risk patients within 48 hours after major abdominal surgery was conducted. Patients' preoperative characteristics, intraoperative management, and outcome were evaluated for associations with AKI using a logistic regression model.

Results: Data from 258 patients were analyzed. Thirty-one patients (12\%) developed AKI, reaching the AKIN stage 1. No patient reached an AKIN stage higher than 1. AKI patients were older ( 75.2 vs 70.2 years; $P=0.0113$ ) and had a higher body mass index ( $26.5 \mathrm{vs} 25.1 \mathrm{~kg} / \mathrm{m}^{2}$ ). In addition, AKI patients had a significantly longer ICU length of stay ( 3.4 vs 2.4 days; $P=.0017$ ). Creatinine levels of AKI patients increased significantly compared to the preoperative levels at $24(P=.0486), 48(P=.0011)$ and 72 hours $(P=.0055)$, while after 72 hours it showed a downwards trend. At ICU discharge, 28 out of 31 patients (90.3\%) recovered preoperative levels.

Multivariate analysis identified age (OR 1.088; $P=.002)$ and BMI (OR 1.124; $P=.022$ ) as risk factors for AKI development. Moreover, AKI development was an independent risk factor for ICU stays longer than 48 hours (OR $2.561 ; P=.019)$.

Conclusions: Mild AKI is a not rare complication in high-risk patients undergoing major abdominal surgery. Although in almost the totality of cases, the indicators of renal function recovered to preoperative levels, postoperative AKI represents a primary risk factor for a prolonged ICU stay.
\end{abstract}

(c) 2016 Elsevier Inc. All rights reserved.

\section{Introduction}

Acute kidney injury (AKI) is a frequent postoperative complication after major surgery $[1,2]$. A recent international cross-sectional study (AKI-EPI) [3], with the aim to analyze the epidemiology of AKI in patients admitted to intensive care unit (ICU), showed that $48.1 \%$ of AKI patients had surgery before ICU admission, confirming its high prevalence in post-surgical population. AKI is associated with longer hospital

\footnotetext{
is Conflicts of Interest: The authors have no conflicts of interest to declare.

* Corresponding author at: Careggi University Hospital, Largo Brambilla 3, 50100, Florence, Italy.

E-mail address: giovanni.zagli@unifi.it (G. Zagli).
}

stays, increased risk for nosocomial infections, and significant burden on costs [4-6]. Its importance as a risk factor for a worse prognosis in noncardiac, non-thoracic, and non-vascular patients has been emphasized $[7,8]$. Over the last 10 years, the diffusion and application of guidelines and recommendations on diagnosis and management of kidney dysfunction has alerted physicians regarding this previously overlooked issue, leading to a significant increase in diagnostic capability and epidemiologic research of patients with AKI [9-12]. Although great attention is focused on kidney function, to date, many aspects of postoperative AKI remain uncertain, and the relationship between perioperative care and renal dysfunction has not been fully addressed [7,13].

The aim of the present study was to evaluate the incidence and predisposing factors of AKI in high-risk patients undergoing major abdominal surgery. 


\section{Methods}

\subsection{Setting and study population.}

All high-risk patients (less than 6 metabolic equivalent (MET) [14], and/or a Revised Cardiac Risk Index for Pre-Operative Risk (RCRI) $\geq 3$ [15] scheduled for major abdominal surgery (colectomy, hepatic resection, pancreatic surgery, esophageal resection, gastric surgery [16]), were electively admitted to the ICU [17-20] and included in the study group. The AKI group included all those patients who met the AKIN criteria during the 48 hours following surgery (any stage) [10]. In order to specifically address the potential impact of surgical and anaesthesiology approaches on renal function, and limit the possibility of $\mathrm{mid} /$ long term confounding factors (eg, sepsis, hemodynamic impairments, or other complications), according to AKI definitions [11] and based on recent literature observations [21], patients included were those who developed AKI within 48 hours after surgery. The following perioperative data were recorded: age, body mass index (BMI), comorbidities (arteriopathy, diabetes, arterial hypertension, ischemic heart disease, chronic obstructive pulmonary disease), surgical technique (open laparotomy, videolaparoscopic, robotic-assisted), surgical time, ICU length of stay (LoS), basal (pre-operative) and daily serum creatinine, urinary output, and fluid balance. The Institutional Ethics Committee approved the study. Informed consent was obtained from all the participants.

\subsection{Intraoperative management}

Intraoperative fluid therapy was set at $5 \mathrm{~mL} / \mathrm{kg}$ per hour of crystalloid solutions (lactate's Ringer or Acetate Ringer) [22] in both open and minimally invasive techniques performed with pneumoperitoneum (no carbon dioxide re-uptake and warming was used). During surgery, the attending anesthetist was free to administer additional fluid boluses. Anesthesia was delivered according to personal preference of the attending anaesthesiologists (total intra-venous or balanced) and adapted to entropy-based neuromonitoring (Entropy, GE Healthcare, Milwaukee, WI). Arterial blood pressure was monitored with radial catheter (20 gauge). Fluid administration was based on pulse pressure variation $(\mathrm{PPV}[\%]=(\mathrm{PPmax}-\mathrm{PPmin}) /$ $[($ PPmax + PPmin $) / 2] * 100 ;$ GE Healthcare; Milwaukee, USA), keeping it under $10 \%$ [23]. Mean blood pressure was maintained above $65 \mathrm{mmHg}$. Central body temperature was monitored with a nasopharyngeal probe and kept at $36.5-37^{\circ} \mathrm{C}$. Neuromuscular block was monitored with the Train of Four ratio (E-NMT Module, GE Healthcare, Milwaukee, U.S.A).

\subsection{Statistical analysis}

The SPSS 21 (SPSS Inc, Chicago, IL) was used for statistical analysis. Continuous variables were analyzed with the two-tailed MannWhitney $U$ test. Categorical variables were studied using Fisher exact test. A 2 -sided $P<.05$ was considered to be statistically significant. Continuous variables are expressed as mean (SD). In order to define the possible clinical factors associated with the development of AKI, a multivariable logistic regression model (backward selection) was adopted (covariates were retained in the model if the $P$ value was $<.1$ ). The odds ratio (OR) and 95\% confidence interval $(\mathrm{CI})$ were computed. The ICU LoS was dichotomized at 48 hours in consideration that patients included were admitted in ICU for postoperative management and monitoring (thus foreseeing a $\operatorname{LoS} \leq 48 \mathrm{~h}$ ).

Expecting proportion of AKI patient in our population was $7.5 \%$ [1], we estimated to enroll at least 192 patients to guarantee a margin of error, equal or less than $5 \%$ (5\%-15\%) using a $95 \%$ confidence level.

\section{Results}

During a 9-month study period, a total of 899 patients underwent general surgery: among them, 258 met the inclusion criteria and were enrolled in the study (Fig. 1). Baseline and clinical characteristics of the patients are summarized in Table 1. Overall, 31 patients (12\%) developed AKI, reaching AKIN stage 1 during the first 48 hours. Two patients developed AKI after the first 48 hours and, according to study protocol, were excluded: one patient reached AKIN 1 stage at postoperative day 5 and the second one reached AKIN 1 stage on day 7 .

The mean age of AKI patients resulted significantly higher than the NO-AKI group (75.2 (8.9) vs $70.2(11.2)$ years; $P=.0113)$, as well as BMI (26.5(3.4) vs $\left.25.1(4.4) \mathrm{Kg} / \mathrm{m}^{2} ; P=.0067\right)$. Surgical approaches are summarized in Fig. 1, including a group with both videolaparoscopic and robotic-assisted techniques (both performed with pneumoperitoneum). AKI patients had significantly longer ICU LoS than NO-AKI group (3.4 (1.9) vs 2.4 (1.7) days; $P=.0017$ ) (Table 1 ).

Fig. 2 shows the serum creatinine level trends: whereas the preoperative mean creatinine levels were similar between the two groups, AKI patients showed significantly higher levels during the study period than NO-AKI patients (Mann-Whitney test, $P<.001$ ). Increase in creatinine was statistically significant at 24 hours $(P=.0486)$, at 48 hours $(P=$ $.0011)$, and at 72 hours $(P=.0055)$. After 72 hours the creatinine levels showed a downward trend, and, at the end of monitoring (day 5 after surgery), 28 out of 31 patients (90.3\%) recovered preoperative levels of creatinine. In order to evaluate difference in creatinine trend from the third day onwards a generalized estimating equation linear regression model was used. The difference in slope between AKI and NO-AKI groups was not statistically significant $(P=.2929)$. Three patients did not recover baseline creatinine after the first 5 postoperative days but were eventually discharged from the hospital with a creatinine level insignificantly different to the preoperative one. Among groups, neither fluid balance (Fig. 3) nor urinary output (Fig. 4) differed significantly during the study period.

Table 2 summarizes multiple logistic regression analyses, which included age, gender, BMI, surgical technique and duration, and comorbidities. Age (OR 1.088, 95\% CI 1.032-1.146, $P=.002$ ) and BMI (OR $1.124,95 \%$ CI 1.017-1.242, $P=.022$ ) were independent risk factors for early postoperative AKI development, whereas videolaparoscopic surgical technique resulted an independent protective factor for AKI (OR 0.264, 95\% CI 0.093-0.746, $P=.012$ ).

Based on the observation that AKI patients showed a significantly longer ICU LoS, a multivariate analysis was also performed to verify if AKI could be an independent risk factor for a longer ICU stay. The model included age, gender, BMI, surgical technique, operation duration, comorbidities: AKI was associated with a significant increased risk to have an ICU stay longer than 48 hours (OR 2.561, 95\% CI 1.1695.068; $P=.019)$.

\section{Discussion}

The main finding of this study was that a consistent incidence of early AKI (within 48 hours) after major abdominal surgery in highrisk patients (12\%) occurs. Interestingly, in this cohort only 2 patients had delayed AKI (later than 48 hours post-operatively), showing that, in the context of uncomplicated high risk surgery, delayed AKI is a rare occurrence. Despite the fact that in this population early AKI severity never exceeded stage 1 , and most of them (90.3\%) recovered before ICU discharge, this study clearly shows that AKI is a strong predisposing factor for prolonged ICU stay (OR 2.561). This result is even more interesting considering that we designed the study excluding many potential confounding factors (eg, need of transfusion) to focus the attention on surgical population without factors known to increase AKI incidence.

A recent large retrospective study on about 160,000 patients undergoing major surgery (about 45,000 of them underwent general surgery) [24] showed an incidence of stage 1 AKI of $9.4 \%$, similar to the 


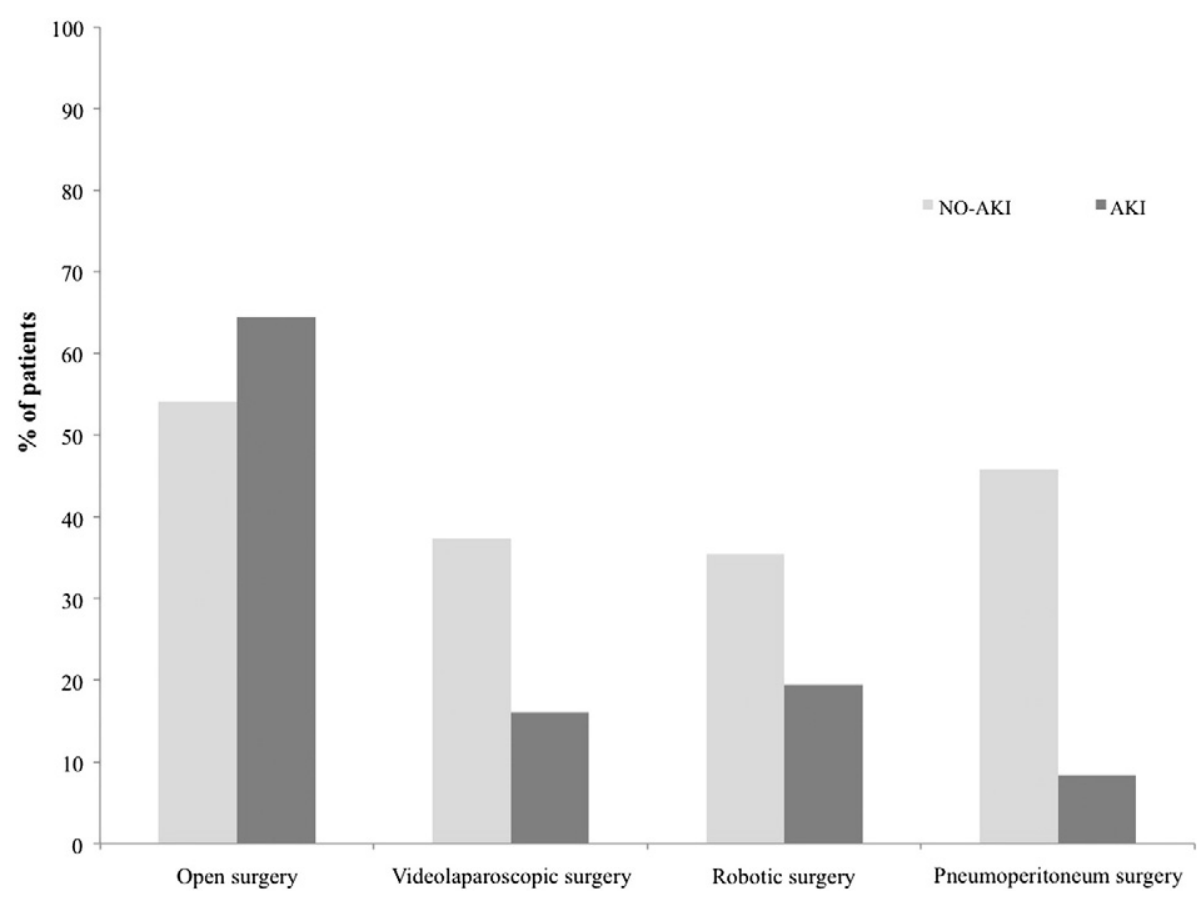

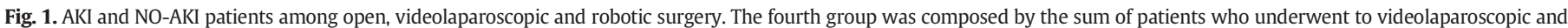
robotic surgery ("pneumoperitoneum group").

percentage found in our sample, and confirmed that the development of stage 1 AKI represents a primary risk factor for a prolonged ICU (and hospital) stay. In their retrospective study, which included long-term observations, Grams et al. showed also that AKI patients had a higher risk of hospital readmission, mortality, and development of end-stage renal failure [24], proportionally with any AKI stages. The incidence of AKI in our population was also similar to another recent systematic review [25], in which Authors calculated a post-operative AKI incidence of $13.4 \%$ in patients undergoing to abdominal surgery.

The other 2 findings of our study were the association of AKI development with age and BMI. Age is a well-known risk factor for renal function impairment [26], since the kidney function reserve decreases with age and, due to this natural phenomenon, even minor injury can produce function impairment. Obesity is known to be a "generic" risk factor for numerous pathologies, due to its impact on metabolism and microcirculation. However, it has recently been shown that a high BMI is an independent risk factor for AKI both in general surgical [27] and in cardiac surgical patients [28]. Moreover, the study of Grams and Colleagues [24] confirmed that both age and BMI are independent risk factors for postoperative AKI development.

Although urine output is one of the criteria for AKI diagnosis together with creatinine increase, in our cohort of patients, diuresis showed a different behavior and trend between AKI and NO-AKI groups. This may be explained by the use of (a higher dose of) diuretics, very common in most critically ill patients who are those receiving the highest fluid amount and consequently are at highest risk for fluid accumulation

Table 1

Baseline and clinical characteristics of the overall population, AKI and NO-AKI patients

\begin{tabular}{|c|c|c|c|c|}
\hline & Overall & NO-AKI & AKI & $P$ \\
\hline Number (\%) & 258 & $227(88 \%)$ & $31(12 \%)$ & \\
\hline Male sex, N (\%) & $151(58.5 \%)$ & $130(57.3 \%)$ & $21(67.7 \%)$ & .3325 \\
\hline Age (years) & $70.8 \pm 11.1$ & $70.2 \pm 11.2$ & $75.2 \pm 8.9$ & .0113 \\
\hline BMI & $25.2 \pm 4.3$ & $25.1 \pm 4.4$ & $26.5 \pm 3.4$ & .0067 \\
\hline \multicolumn{5}{|l|}{ Surgical technique, $\mathrm{N}(\%)$} \\
\hline Open & $143(55.4 \%)$ & $123(54.2 \%)$ & $20(64.5 \%)$ & \\
\hline Videolaparoscopic & $90(34.9 \%)$ & $85(37.4 \%)$ & $5(16.1 \%)$ & .0507 \\
\hline Robotic & $25(9.7 \%)$ & $19(8.4 \%)$ & $6(19.4 \%)$ & .2303 \\
\hline Videolaparoscopy + robotic (pneumoperitoneum) & $115(44.6 \%)$ & $104(45.8 \%)$ & $11(35.5 \%)$ & .3372 \\
\hline Surgical time (min) & $265 \pm 134$ & $259 \pm 126$ & $309 \pm 177$ & .1608 \\
\hline Open & $264 \pm 125$ & $264 \pm 121$ & $267 \pm 143$ & .9528 \\
\hline Videolaparoscopic & $246 \pm 139$ & $332 \pm 261$ & $241 \pm 130$ & .4573 \\
\hline Robotic & $339 \pm 147$ & $428 \pm 176$ & $311 \pm 130$ & .1909 \\
\hline Videolaparoscopy + robotic (pneumoperitoneum) & $266 \pm 145$ & $384 \pm 215$ & $254 \pm 132$ & .0264 \\
\hline ICU length of stay (days) & $2.5 \pm 1.7$ & $2.4 \pm 1.7$ & $3.4 \pm 1.9$ & $<.0017$ \\
\hline Open & $2.8 \pm 1.9$ & $2.6 \pm 1.9$ & $3.6 \pm 1.8$ & .0154 \\
\hline Videolaparoscopic & $2.1 \pm 1.5$ & $3 \pm 2.4$ & $2.1 \pm 1.4$ & .4375 \\
\hline Robotic & $2.6 \pm 1.6$ & $3 . \overline{2} \pm 1.9$ & $2.4 \pm 1.5$ & .4044 \\
\hline Videolaparoscopy + robotic (pneumoperitoneum) & $2.2 \pm 1.5$ & $3.1 \pm 2$ & $2.1 \pm 1.4$ & .1171 \\
\hline
\end{tabular}

Continuous data are expressed as mean \pm standard deviation (SD).

Percentages of patients with male sex and surgical techniques having AKI and NO-AKI are referred to total population of AKI and NO-AKI populations, respectively.

Videolaparoscopic and robotic (and videolaparoscopic + robotic) techniques were compared to the open technique.

Statistical analysis: two-tailed Mann-Whitney test and two tails Fisher's exact test. $P$ significant if $<.05$.

Abbreviations: BMI, body mass index; ICU, Intensive Care Unit. 


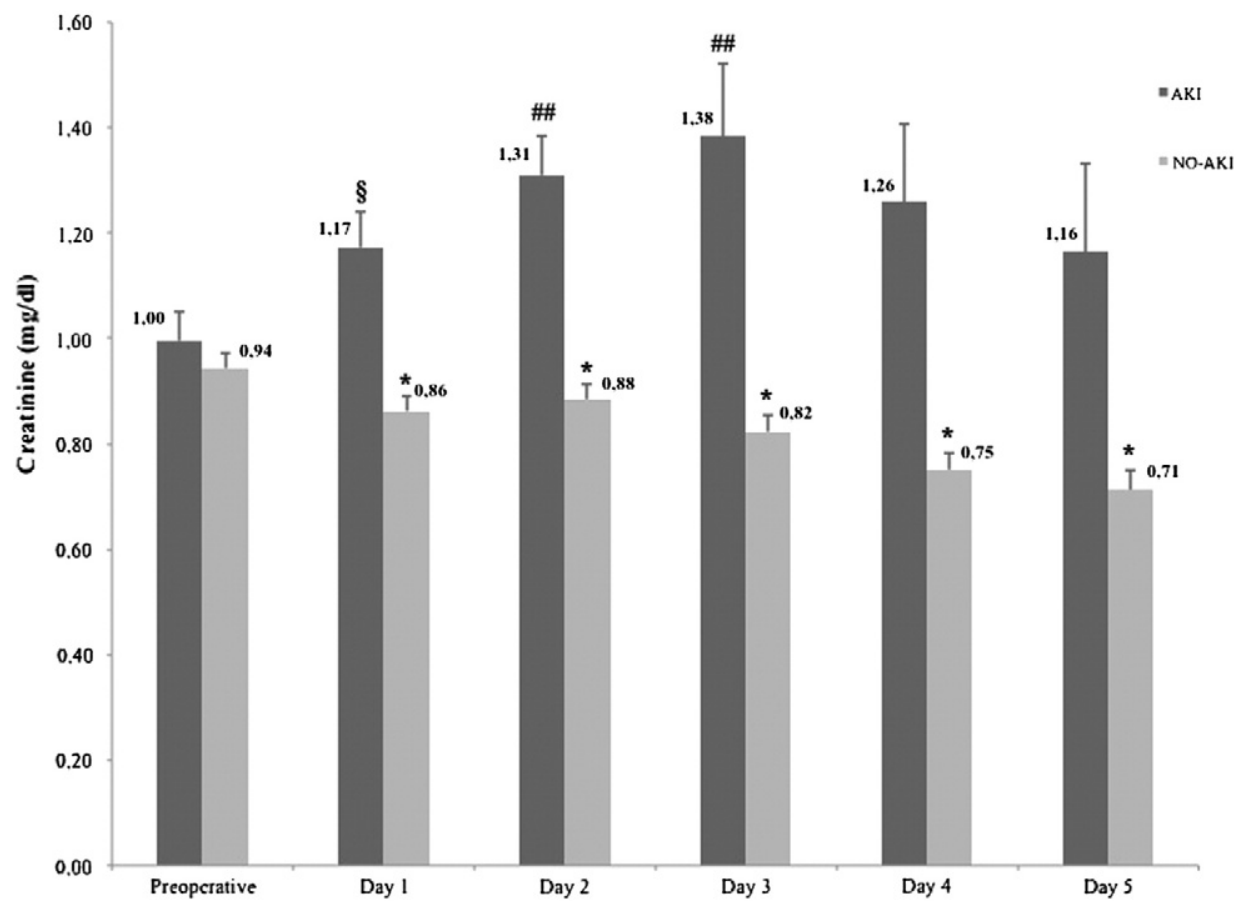

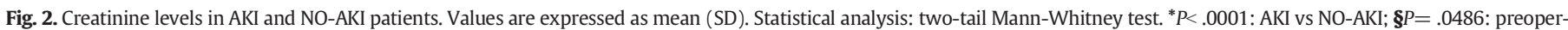
ative vs day 1 in $A K I$ patients; $\# P=.0011$ : preoperative vs day 2 in AKI patients; $\# \# P=.0055$ : preoperative vs day 3 in AKI patients.

and AKI. Although diuretics are not recommended by current guidelines for AKI prevention or treatment [29], they have a pivotal role for volume management in order to avoid fluid overload and weight gain especially when renal function is reduced [30].

The best postoperative management in order to avoid AKI has been investigated by many authors. In patients undergoing elective colorectal resection, Brandstrup and coauthors showed that a restricted perioperative intravenous fluid regimen (aimed to avoid body weight gain) was associated with a decrease in overall post-operative complications, including renal failure, even if Brandstrup and coauthors limited the analysis of AKI incidence to patients who required dialysis [31]. More recently, Prowle and coworkers proposed that fluid restriction and vasoactive support might obtain better results in terms of renal function protection [32]. However, the better fluid administration strategy is still highly debated since fluid loading always represents a "double-edged sword" [22,33,34].

Intriguingly, our data suggested a potential protective role of videolaparoscopic surgery, despite increased abdominal pressure due to pneumoperitoneum (Table 1). On one hand, increased intraabdominal pressure may have direct and indirect negative effects on kidney function due to several mechanisms: direct compression on the renal parenchyma; reduced renal blood flow due to cardiac output (caused by reduced venous return); increase in blood $\mathrm{CO}_{2}$; and increased vascular resistance. All these effects can result in a reduced

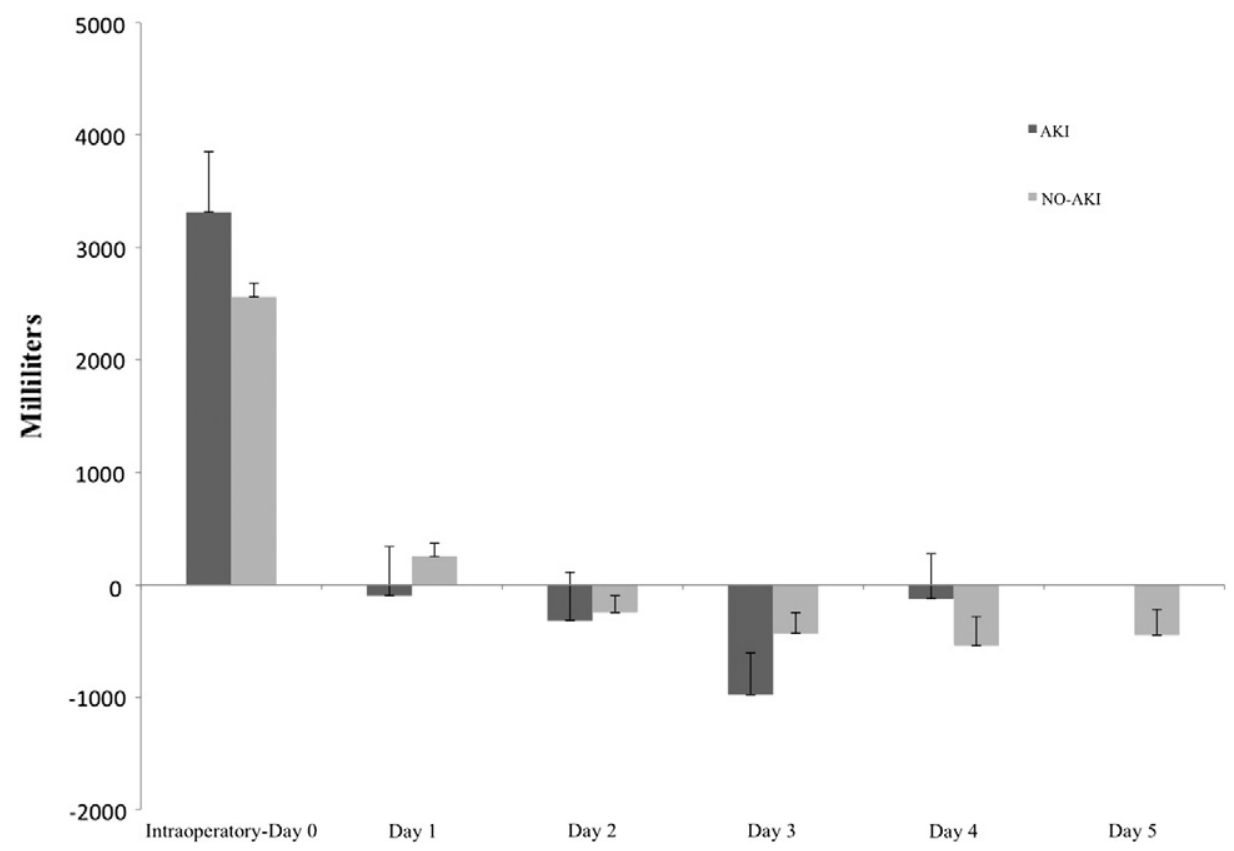

Fig. 3. Fluid balance in AKI and NO-AKI patients from intraoperative time until ICU discharge. Values are expressed as mean (SD). 


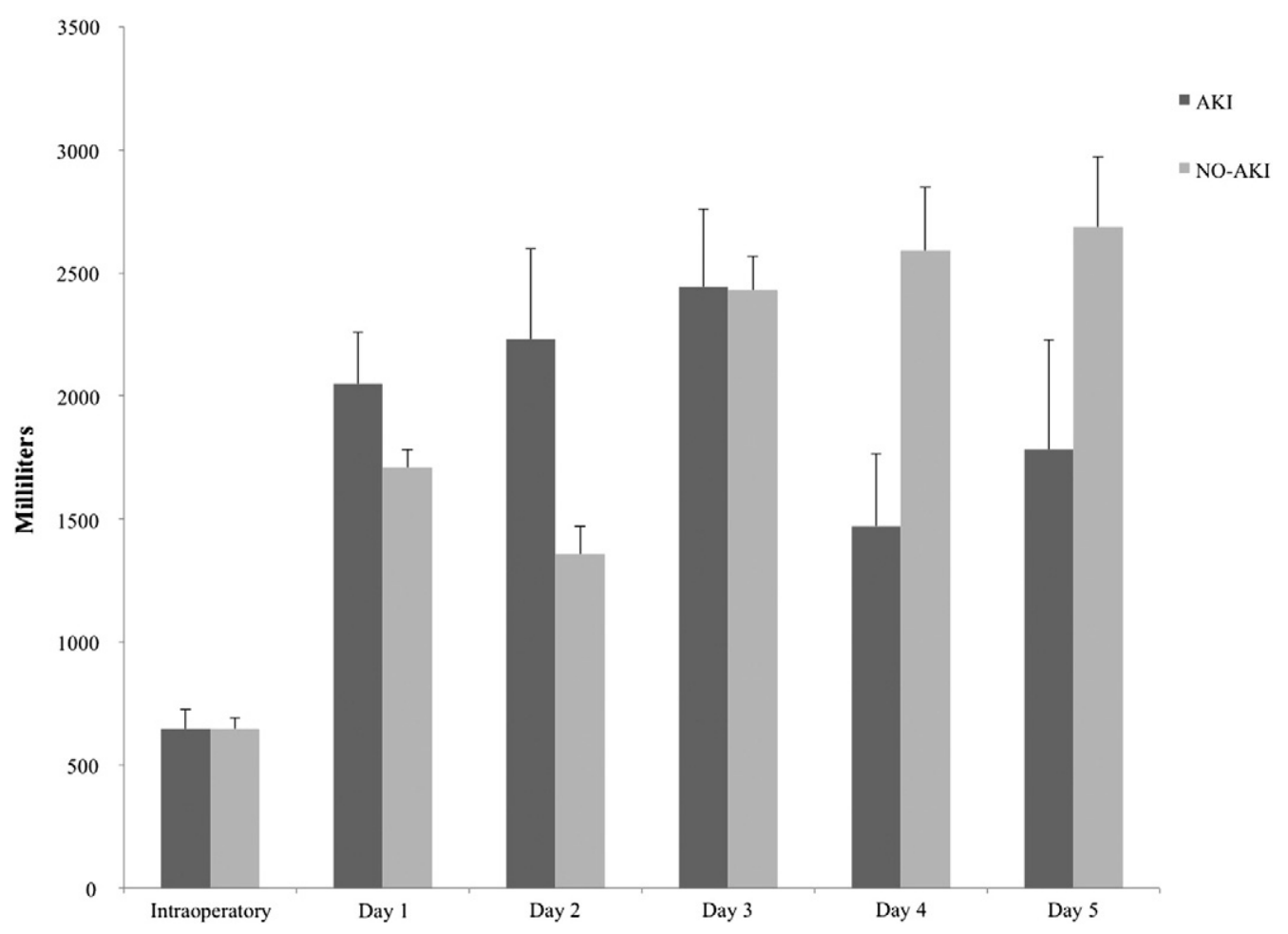

Fig. 4. Urinary output in AKI and NO-AKI patients from intraoperative time until ICU discharge. Values are expressed as mean (SD).

glomerular filtration rate $[11,35]$. In addition, alterations in systemic and renal hemodynamic may activate the renin-angiotensin-aldosterone system, which further increases renal vascular resistance [36,37]. On the other hand, we should consider that $12 \mathrm{mmHg}$ is the highest pressure usually reached during robotic or laparoscopic surgery, which represents the threshold for abdominal hypertension definition [38]. Importantly, surgical stress and tissue trauma, clearly more intense in open than in minimally invasive surgery, is thought to be associated with the postoperative inflammation [39]. Inflammatory mediators are important components of both the initiation and extension of kidney injury [7]. Therefore, laparoscopic and robotic surgery may exert a protective role for kidney function by limiting inflammation although this aspect need to be confirmed in studies focused on.

\section{Conclusions}

In conclusion, early AKI is a relatively frequent occurrence in highrisk patients undergoing major abdominal surgery. We found a significant impact of AKI on ICU LoS, especially in elderly and obese patients. This should encourage clinicians to focus their attention on such specific patients given that, even if not severe, early renal dysfunction in the post-operative phase has clinically relevant effects.

Table 2

Multivariate regression analysis for predictors of AKI development (backward selection; entry criterion of $P<.05$ and a removal criterion of $P>.1$ )

\begin{tabular}{llll}
\hline & OR & $95 \% \mathrm{CI}$ & $P$ \\
\hline Age & 1.088 & $1.032-1.146$ & .002 \\
BMI & 1.124 & $1.017-1.242$ & .022 \\
Videolaparoscopic surgery & 0.264 & $0.093-0.746$ & .012 \\
\hline
\end{tabular}

Abbreviations: OR, odds ratio; $\mathrm{CI}$, confidence interval.

\section{References}

[1] Abelha FJ, Botelho M, Fernandes V, Barros H. Determinants of postoperative acute kidney injury. Crit Care 2009;13(3):R79.

[2] Sear JW. Kidney dysfunction in the postoperative period. Br J Anaesth 2005;95(1): 20-32.

[3] Hoste EA, Bagshaw SM, Bellomo R, Cely CM, Colman R, Cruz DN, et al. Epidemiology of acute kidney injury in critically ill patients: the multinational AKI-EPI study. Intensive Care Med 2015;41(8):1411-23.

[4] Pruchnicki MC, Dasta JF. Acute renal failure in hospitalized patients: part I. Ann Pharmacother 2002;36(7-8):1261-7.

[5] Pruchnicki MC, Dasta JF. Acute renal failure in hospitalized patients: part II. Ann Pharmacother 2002;36(9):1430-42.

[6] Vincent JL, Bota DP, De Backer D. Epidemiology and outcome in renal failure. Int J Artif Organs 2004;27(12):1013-8.

[7] Romagnoli S, Ricci Z. Postoperative acute kidney injury. Minerva Anestesiol 2015; 81(6):684-96.

[8] Schmid M, Dalela D, Tahbaz R, Langetepe J, Randazzo M, Dahlem R, et al. Novel biomarkers of acute kidney injury: evaluation and evidence in urologic surgery. World J Nephrol 2015;4(2):160-8.

[9] Bellomo R, Ronco C, Kellum JA, Mehta RL, Palevsky P. Acute renal failure - definition, outcome measures, animal models, fluid therapy and information technology needs: the second international consensus conference of the acute dialysis quality initiative (ADQI) group. Crit Care 2004;8(4):R204-12.

[10] Mehta RL, Kellum JA, Shah SV, Molitoris BA, Ronco C, Warnock DG, et al. Acute kidney injury network: report of an initiative to improve outcomes in acute kidney injury. Crit Care 2007;11(2):R31.

[11] Group KAGW. Kidney disease: improving global outcomes (KDIGO) acute kidney injury work group. KDIGO clinical practice guideline for acute kidney injury. Kidney Int Suppl 2012;2:1-138.

[12] Hoste EA, De Corte W. Implementing the kidney disease: improving global outcomes/acute kidney injury guidelines in ICU patients. Curr Opin Crit Care 2013; 19(6):544-53.

[13] Sun LY, Wijeysundera DN, Tait GA, Beattie WS. Association of Intraoperative Hypotension with acute kidney injury after elective noncardiac surgery. Anesthesiology 2015;123(3):515-23.

[14] James S, Jhanji S, Smith A, O'Brien G, Fitzgibbon M, Pearse RM. Comparison of the prognostic accuracy of scoring systems, cardiopulmonary exercise testing, and plasma biomarkers: a single-Centre observational pilot study. Br J Anaesth 2014;112(3): 491-7.

[15] Lee TH, Marcantonio ER, Mangione CM, Thomas EJ, Polanczyk CA, Cook EF, et al. Derivation and prospective validation of a simple index for prediction of cardiac risk of major noncardiac surgery. Circulation 1999;100(10):1043-9.

[16] Banz VM, Jakob SM, Inderbitzin D. Review article: improving outcome after major surgery: pathophysiological considerations. Anesth Analg 2011; 112(5):1147-55. 
[17] Khuri SF, Henderson WG, DePalma RG, Mosca C, Healey NA, Kumbhani DJ. Determinants of long-term survival after major surgery and the adverse effect of postoperative complications. Ann Surg 2005;242(3):326-41 [discussion 341-323].

[18] Pearse RM, Harrison DA, James P, Watson D, Hinds C, Rhodes A, et al. Identification and characterisation of the high-risk surgical population in the United Kingdom. Crit Care 2006;10(3):R81.

[19] Jhanji S, Thomas B, Ely A, Watson D, Hinds CJ, Pearse RM. Mortality and utilisation of critical care resources amongst high-risk surgical patients in a large NHS trust. Anaesthesia 2008;63(7):695-700.

[20] Gruppo Italiano per la Valutazione degli Interventi in Terapia Intensiva - Istituto Mario Negri. http://www.giviti.marionegri.it/StART.asp.

[21] Kim M, Brady JE, Li G. Variations in the risk of acute kidney injury across intraabdominal surgery procedures. Anesth Analg 2014;119(5):1121-32.

[22] Della Rocca G, Vetrugno L, Tripi G, Deana C, Barbariol F, Pompei L. Liberal or restricted fluid administration: are we ready for a proposal of a restricted intraoperative approach? BMC Anesthesiol 2014;14:62.

[23] Salzwedel C, Puig J, Carstens A, Bein B, Molnar Z, Kiss K, et al. Perioperative goaldirected hemodynamic therapy based on radial arterial pulse pressure variation and continuous cardiac index trending reduces postoperative complications after major abdominal surgery: a multi-center, prospective, randomized study. Crit Care 2013;17(5):R191.

[24] Grams ME, Sang Y, Coresh J, Ballew S, Matsushita K, Molnar MZ, et al. Acute kidney injury after major surgery: a retrospective analysis of veterans health administration data. Am J Kidney Dis 2015.

[25] O'Connor ME, Kirwan CJ, Pearse RM, Prowle JR. Incidence and associations of acute kidney injury after major abdominal surgery. Intensive Care Med 2015.

[26] Martensson J, Bellomo R. Perioperative renal failure in elderly patients. Curr Opin Anaesthesiol 2015;28(2):123-30.

[27] Buehler L, Fayfman M, Alexopoulos AS, Zhao L, Farrokhi F, Weaver J, et al. The impact of hyperglycemia and obesity on hospitalization costs and clinical outcome in general surgery patients. J Diabetes Complicat 2015;29(8):1177-82.
[28] O'Sullivan KE, Byrne JS, Hudson A, Murphy AM, Sadlier DM, Hurley JP. The effect of obesity on acute kidney injury after cardiac surgery. J Thorac Cardiovasc Surg 2015;150(6):1622-8

[29] Kidney International.

[30] Grams ME, Estrella MM, Coresh J, Brower RG, Liu KD. Fluid balance, diuretic use, and mortality in acute kidney injury. Clin J Am Soc Nephrol 2011;6(5):966-73.

[31] Brandstrup B, Tonnesen H, Beier-Holgersen R, Hjortso E, Ording H, Lindorff-Larsen K, et al. Effects of intravenous fluid restriction on postoperative complications: comparison of two perioperative fluid regimens: a randomized assessor-blinded multicenter trial. Ann Surg 2003;238(5):641-8.

[32] Prowle JR, Chua HR, Bagshaw SM, Bellomo R. Clinical review: volume of fluid resuscitation and the incidence of acute kidney injury - a systematic review. Crit Care 2012;16(4):230.

[33] Bundgaard-Nielsen M, Secher NH, Kehlet H. 'liberal' vs 'restrictive' perioperative fluid therapy-a critical assessment of the evidence. Acta Anaesthesiol Scand 2009; 53(7):843-51.

[34] Kellum JA. Critical care nephrology. Crit Care Clin 2015;31(4):xiii-xv.

[35] Harman PK, Kron IL, McLachlan HD, Freedlender AE, Nolan SP. Elevated intraabdominal pressure and renal function. Ann Surg 1982;196(5):594-7.

[36] Richards WO, Scovill W, Shin B, Reed W. Acute renal failure associated with increased intra-abdominal pressure. Ann Surg 1983;197(2):183-7.

[37] Sugrue M, Buist MD, Hourihan F, Deane S, Bauman A, Hillman K. Prospective study of intra-abdominal hypertension and renal function after laparotomy. $\mathrm{Br} J$ Surg 1995; 82(2):235-8.

[38] Kirkpatrick AW, Roberts DJ, De Waele J, Jaeschke R, Malbrain ML, De Keulenaer B, et al. Intra-abdominal hypertension and the abdominal compartment syndrome: updated consensus definitions and clinical practice guidelines from the world Society of the Abdominal Compartment Syndrome. Intensive Care Med 2013;39(7): 1190-206.

[39] Okholm C, Goetze JP, Svendsen LB, Achiam MP. Inflammatory response in laparoscopic vs open surgery for gastric cancer. Scand J Gastroenterol 2014;49(9):1027-34. 